\title{
Medical Image Recognition of Heart Regions by Deep Multi-Layered GMDH-Type Neural Network Using Principal Component-Regression Analysis
}

\author{
Tadashi Kondo \\ Graduate School of Health Sciences, Tokushima University \\ 3-18-15 Kuramoto-cho Tokushima 770-8509 Japan \\ Email:kondomedsci@gmail.com \\ Junji Ueno \\ Graduate School of Health Sciences, Tokushima University \\ 3-18-15 Kuramoto-cho Tokushima 770-8509 Japan \\ Shoichiro Takao \\ Graduate School of Health Sciences, Tokushima University \\ 3-18-15 Kuramoto-cho Tokushima 770-8509 Japan
}

\begin{abstract}
In this study, a deep Group Method of Data Handling (GMDH)-type neural network using principal componentregression is applied to the medical image recognition of the heart regions. The deep GMDH-type neural network algorithm can organize the neural network architecture with many hidden layers fitting the complexity of the nonlinear systems so as to minimize the prediction error criterion defined as AIC (Akaike's Information Criterion) or PSS (Prediction Sum of Squares). This algorithm is applied to the medical image recognition of the heart regions and it is shown that this algorithm is useful for the medical image recognition of the heart regions because deep neural network architecture is automatically organized using the principal component-regression analysis from the medical images of the heart regions.
\end{abstract}

Keywords: Deep neural networks, GMDH, Medical image recognition, Evolutionary computation

\section{Introduction}

The deep Group Method of Data Handling (GMDH)-type neural networks and their applications have been proposed in our early works $^{1 \sim 4}$. Deep GMDH-type neural networks can automatically organize neural network architecture by heuristic selforganization method ${ }^{5,6}$, which is a type of evolutionary computation. In our early works ${ }^{3,4}$, we applied the conventional GMDH-type neural network algorithms to the medical image recognition of the heart regions, and these GMDH-type neural network algorithms use the stepwise regression analysis as the learning algorithm of the neural network and the multi-colinearity of the weights which is occurred in heuristic self-organization method of the GMDH algorithms, cannot be eliminated completely. The prediction values of the neural network become unstable and these values are not accurate when the multi-colinearity occurs in the learning calculation of the neural networks. Furthermore, these conventional GMDH-type neural network algorithms cannot select automatically optimum neural network architectures from three types of neural network architectures such as sigmoid function neural network, radial basis function (RBF) neural network and polynomial neural network. 
In this study, a deep GMDH-type neural network algorithm using the principal component-regression analysis is developed and applied to the medical image recognition of the heart regions. In this algorithm, the principal component-regression analysis is used as the learning algorithm of the neural network and the stable and accurate prediction values are obtained. Furthermore, optimum neural network architecture is automatically selected from three types of neural network architectures such as sigmoid function neural network, radial basis function (RBF) neural network and polynomial neural network so as to minimize the prediction error criterion defined as AIC (Akaike's Information Criterion) ${ }^{7}$ or PSS (Prediction Sum of Squares $)^{8}$. This algorithm is applied to the medical image recognition of the heart regions and results show that the deep GMDH-type neural network algorithm is useful for the medical image recognition of the heart regions.

\section{Heuristic self-organization ${ }^{5,6}$}

The architectures of the GMDH-type neural networks are automatically organized using the heuristic self-organization method. The heuristic selforganization method is constructed by the following five procedures.

\section{(1) Separating original data into training and test sets.}

The original data are separated into training and test sets. The training data are used for estimating the parameters of the partial descriptions which describe the partial relationships of the nonlinear system. The test data are used for organizing the complete description which describes the complete relationships between the input and output variables of the nonlinear system.

\section{(2) Generating the combinations of the input variables in each layer.}

All combinations of two input variables $\left(x_{i}, x_{j}\right)$ are generated in each layer. The number of combinations is $P ! /((p-2) ! 2 !)$. Here, $p$ is the number of input variables.

\section{(3) Calculating the partial descriptions.}

For each combination, the partial descriptions of the nonlinear system are calculated by applying the regression analysis to the training data. The output variables of the partial descriptions are called as intermediate variables.

\section{(4) Selecting the intermediate variables.}

The $L$ intermediate variables which give the $L$ smallest test errors calculated using the test data, are selected from the generated intermediate variables. The selected $L$ intermediate variables are set to the input variables of the next layer. The same procedures from procedure (2) to (4) are iterated and the multi-layered architecture is organized.

\section{(5) Stopping the multi-layered iterative computation.}

When the errors of the test data in each layer stop decreasing, the iterative computation is terminated. Finally, the complete description of the nonlinear system is constructed by using the partial descriptions generated in each layer.

Heuristic self-organization method is a type of evolutionary computation.

\section{Deep multi-layered GMDH-type neural network}

Fig.1 shows the architecture of the deep multilayered GMDH-type neural network. This neural network is organized by heuristic self-organization method. Procedures for determining architecture of deep GMDH-type neural network conform to the following:

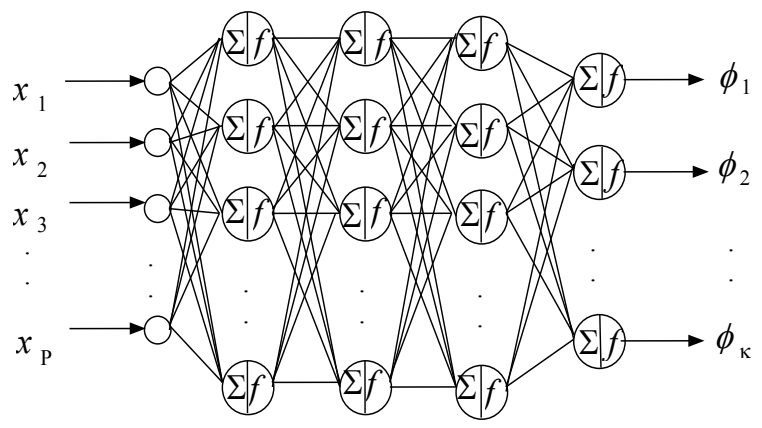

Fig.1 Architecture of the deep GMDH-type neural network 


\subsection{The first layer}

$$
u_{j}=x_{j} \quad(j=1,2, \ldots, p)
$$

where $x_{j}(j=1,2, \ldots, p)$ are input variables of the nonlinear system, and $p$ is the number of input variables.

\subsection{The second layer}

All combinations of $r$ input variables are generated, and $r$ is the number of input variables in each combination. For each combination, optimum neuron architectures are automatically selected from the first and second type neuron architectures. Furthermore, deep GMDH-type neural network algorithm can select optimum neuron architecture from three types of neuron architectures such as sigmoid function neuron, RBF neuron and polynomial neuron. Neuron architectures of the first and second type neurons in each neural network architecture are shown as follows.

\section{(1) Sigmoid function neural network}

\section{1) The first type neuron}

$\Sigma$ : (Nonlinear function)

$$
z_{k}=w_{1} u_{i}+w_{2} u_{j}+w_{3} u_{i} u_{j}+w_{4} u_{i}^{2}+w_{5} u_{j}^{2}-w_{0} \theta_{I}
$$

$f:$ (Nonlinear function)

$$
y_{k}=\frac{1}{1+e^{\left(-z_{k}\right)}}
$$

2) The second type neuron

$\Sigma$ : (Linear function)

$$
z_{k}=w_{1} u_{1}+w_{2} u_{2}+w_{3} u_{3}+\cdots+w_{r} u_{r}-w_{0} \theta_{1} \quad(r<p)
$$

$f:$ (Nonlinear function)

$$
y_{k}=\frac{1}{1+e^{\left(-z_{k}\right)}}
$$

\section{(2) Radial basis function neural network}

\section{1) The first type neuron}

$\Sigma$ : (Nonlinear function)

$$
z_{k}=w_{l} u_{i}+w_{2} u_{j}+w_{3} u_{i} u_{j}+w_{4} u_{i}{ }^{2}+w_{5} u_{j}{ }^{2}-w_{0} \theta_{l}
$$

$f:$ (Nonlinear function)

$$
y_{k}=e^{\left(-z_{k}^{2}\right)}
$$

\section{2) The second type neuron}

$\Sigma$ : (Linear function)

$$
z_{k}=w_{1} u_{1}+w_{2} u_{2}+w_{3} u_{3}+\cdots+w_{r} u_{r}-w_{0} \theta_{l}(r<p)
$$

$f:$ (Nonlinear function)

$$
y_{k}=e^{\left(-z_{k}^{2}\right)}
$$

(3) Polynomial neural network

1) The first type neuron

$\Sigma$ : (Nonlinear function)

$z_{k}=w_{1} u_{i}+w_{2} u_{j}+w_{3} u_{i} u_{j}+w_{4} u_{i}^{2}+w_{5} u_{j}^{2}-w_{0} \theta_{l}$

$f:$ (Linear function)

$$
y_{k}=z_{k}
$$

\section{2) The second type neuron}

$\Sigma$ : (Linear function)

$$
z_{k}=w_{1} u_{1}+w_{2} u_{2}+w_{3} u_{3}+\cdots+w_{r} u_{r}-w_{0} \theta_{l}(r<p)
$$

$f:$ (Linear function)

$$
y_{k}=z_{k}
$$

Here, $\theta_{l}=1$ and $w_{i}(i=0,1,2, \ldots)$ are weights between the first and second layer. Value of $r$, which is the number of input variables $(u)$ in each combination, is set to two for the first type neuron and is set to be greater than two and smaller than $p$ for the second type neuron. Here $p$ is the number of input variables $x_{i}(i=1,2, \ldots, p)$.

\subsubsection{Learning procedures of weight $w_{i}$}

First, values of $z_{k}^{* *}$ are calculated for each neural network architecture as follows.

a) Sigmoid function neural network

b) RBF neural network

$$
z_{k}^{* *}=\log _{e}\left(\frac{\phi^{\prime}}{1-\phi^{\prime}}\right)
$$

c) Polynomial neural network

$$
z_{k}^{* *}=\sqrt{-\log _{e} \phi^{\prime}}
$$

$$
z_{k}^{* *}=\phi
$$

where $\phi^{\prime}$ is the normalized output variable. Then the weights $w_{i}(i=0,1,2, \cdots)$ are estimated using the principal component-regression analysis using the input variable (u) and output variable $\left(z^{* *}\right)$ in each layer.

\subsubsection{Principal component-regression analysis ${ }^{9}$}

In the GMDH-type neural network, the multicolinearity is generated in the function $\Sigma$ of the neurons. In this study, the function $\Sigma$ is calculated using the principal component-regression analysis ${ }^{9}$.

In the case of Eq.(2), the estimation procedures of $z_{k}$ are shown as follows. Here, $z_{k}$ is the estimated value using Eq. (2) for the $z^{* *}$ calculated in 3.2.1.

First, orthogonal vector $\underline{v}$ is calculated.

$$
\underline{\boldsymbol{v}}=C \cdot \underline{\boldsymbol{u}}
$$


Here, $\underline{\boldsymbol{v}}=\left(v_{1}, v_{2}, \ldots, v_{5}\right), \underline{\boldsymbol{u}}=\left(u_{i}, u_{j}, u_{i} u_{j}, u_{i}^{2}, u_{j}^{2}\right)$

$\underline{\boldsymbol{v}}$ is orthonormal vectors and $C$ is orthonormal matrix. $C$ is calculated using the following eigenvalue equation.

$$
R \cdot C=C \cdot \Lambda
$$

Here, $R$ is a correlation matrix. Then, variable $z_{k}$ is calculated using orthogonal regression analysis.

$$
\begin{aligned}
z_{k} & =\underline{\boldsymbol{w}}^{\mathbf{T}} \cdot \underline{\boldsymbol{v}} \\
& =w_{1} v_{1}+w_{2} v_{2}+\ldots+w_{5} v_{5}
\end{aligned}
$$

Using the principal component-regression analysis, variable $z_{k}$ in the function $\Sigma$ is calculated without multicolinearity. In Eq.(19), useful principal components are selected so as to minimize AIC or PSS, and the intermediate variables $z_{k}$ are constructed using selected principal components.

For each combination, three neuron architectures which are sigmoid function neuron, RBF neuron and polynomial neuron, are generated and $L$ neurons which minimize AIC or PSS, are selected for each neuron architecture. From these $L$ selected neurons for each neuron architecture, estimation errors of $L$ neurons are calculated. Then, neural network architecture which has minimum estimation error, is selected as deep GMDHtype neural network architecture from three neural network architectures such as sigmoid function neural network, RBF neural network and polynomial neural network.

After the type of deep GMDH-type neural network architecture is selected, output variables $y_{k}$ of $L$ selected neurons are set to input variables of neurons in the third layer.

\subsection{The third and successive layers}

In the second layer, optimum neural network architecture is selected from three neural network architectures. In the third and successive layers, only one neuron architecture, which is sigmoid function neuron or RBF neuron or polynomial neuron, is used for calculation and the same calculation of the second layer is iterated until AIC or PSS values of $L$ neurons with selected neuron architecture, stop decreasing. When iterative calculation is terminated, neural network architecture is produced by $L$ selected neurons in each layer.
By using these procedures, the deep GMDH-type neural network using the principal componentregression analysis is organized. Deep GMDH-type neural network has an ability of self-selecting optimum neural network architecture. Therefore, neural network architecture is automatically selected from three neural network architectures. Furthermore, structural parameters such as the number of layers, the number of neurons in hidden layers and useful input variables are automatically selected to minimize prediction error criterion defined as AIC or PSS.

\section{Application to the medical image recognition of heart regions}

In this study, the heart regions were automatically recognized using the deep GMDH-type neural network and these regions were extracted. The multi-detector row CT (MDCT) images of the heart regions were used in this study.

\subsection{Results of the medical image recognition by the deep GMDH-type neural network}

The MDCT image shown in Fig.2 was used for organizing the neural network. $x$ and $y$ coordinates and the statistics of the image densities in the neighboring regions of the $N \times N$ pixels at the positions of the learning points, are used as the input variables of the neural network. Only five input variables which are the mean, the standard deviation, the variance and $x$ and $y$ coordinates were automatically selected as useful input variables. The output value of the neural network is zero or one. When $N \times N$ pixel region is contained in the heart regions, the neural network set the pixel value at the center of the $N \times N$ pixel region to one and this pixel is shown as the white point. The neural networks were organized when the values of $N$ were from 3 to 10 . It was determined that when $N$ was equal to 4 , the neural network architecture had the smallest recognition error. Five useful neurons were selected in each hidden layer. Fig. 3 shows errors of the three types of neurons in the second layer. The sigmoid function neural network architecture was selected by the deep GMDH-type neural network algorithm. Fig.4 shows the variation of PSS values. PSS values decreased gradually and very 
small PSS values were obtained at the tenth layer. The heart region was recognized using the organized neural network and was extracted automatically. Fig.5 shows the output image of the deep GMDH-type neural network. This output image was processed by the postprocessing analysis. In the post-processing, the small isolated regions were eliminated and the outlines of the heart regions were expanded outside by $N / 2$ pixels. Fig. 6 shows the output image after this processing. In order to check the matching between the original image and the output image of the neural network, the output image was overlapped on the original image of Fig.2. The overlapped image is shown in Fig.7. From Fig.7, we can see that the output image was very accurate. Fig. 8 shows the extracted heart image.

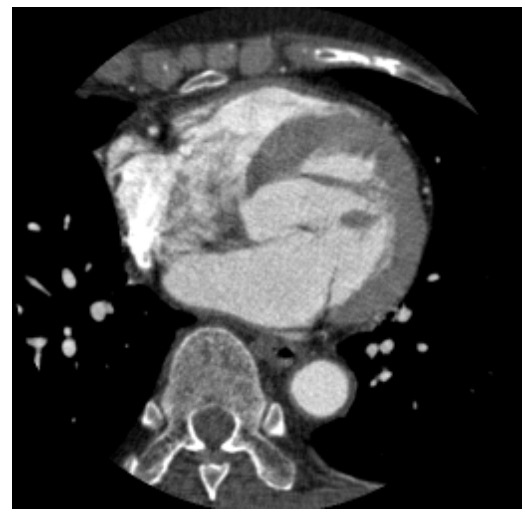

Fig. 2 Original image

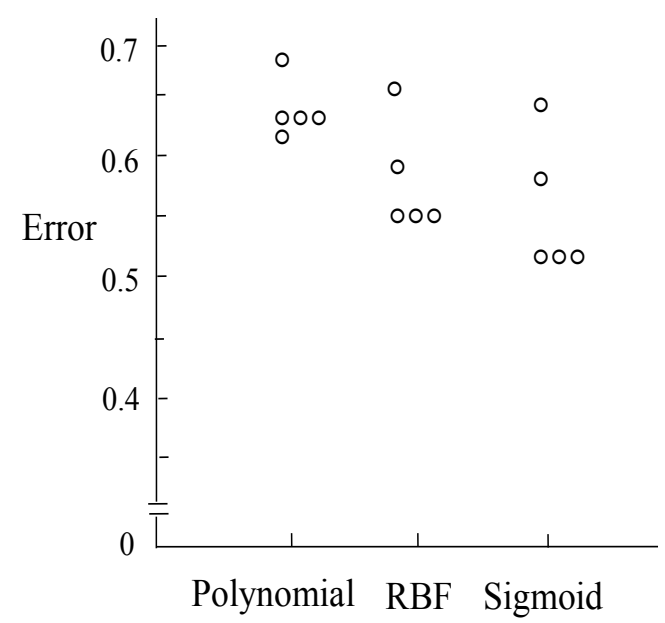

Fig. 3 Errors of three types of neurons

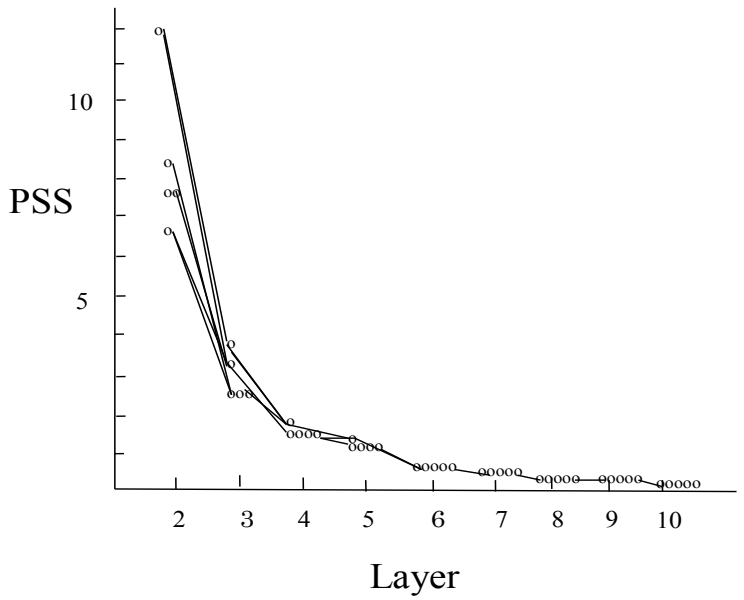

Fig. 4 Variation of PSS in each layer

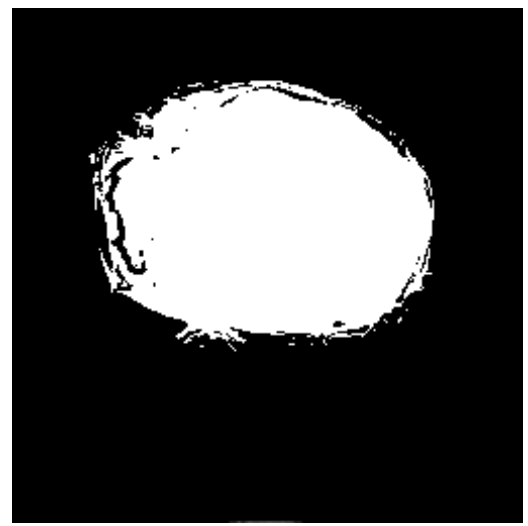

Fig. 5 Output image

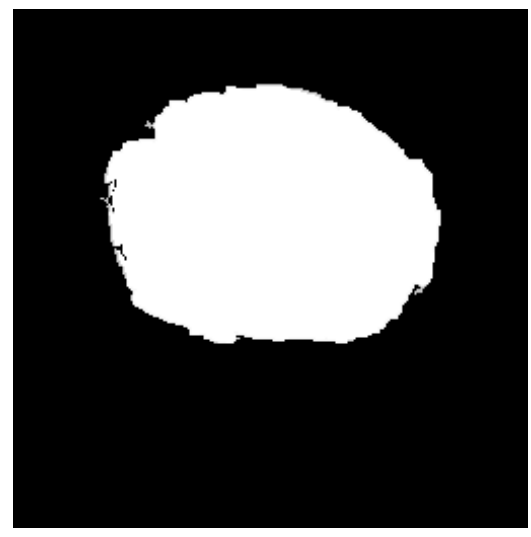

Fig.6 Output image after the post processing 


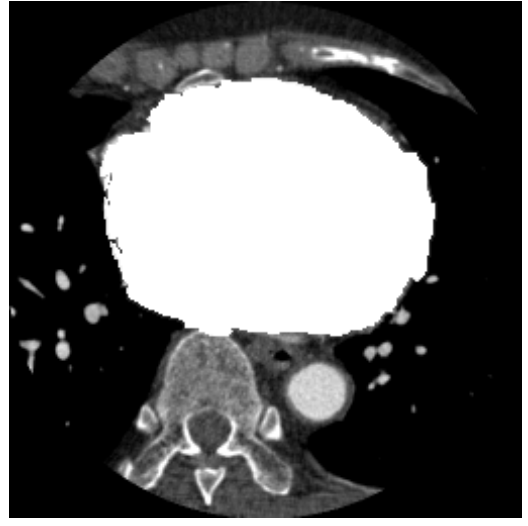

Fig. 7 Overlapped image

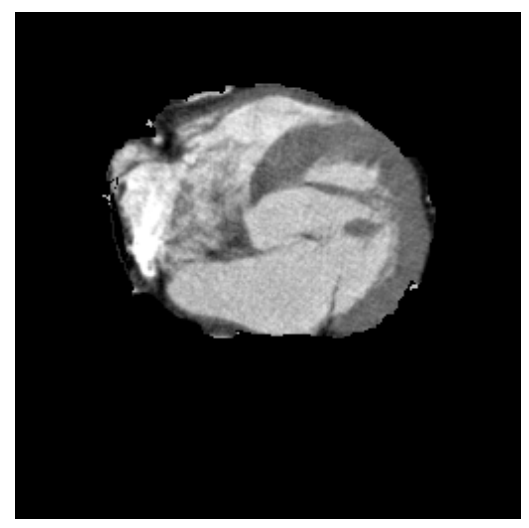

Fig. 8 Extracted image

\subsection{Extraction by the conventional neural network using sigmoid function.}

A conventional neural network trained using the back propagation algorithm was applied to the same recognition problem. The learning calculations of the weights were iterated changing structural parameters such as the number of neurons in the hidden layer and the initial values of the weights. The output images, when the numbers of neurons in the hidden layer $(m)$ are 5, 7 and 9, are shown in Fig.9. These images contain more regions which are not part of the heart and the outlines of the heart are not extracted with required clarity compared with the output images obtained using the deep GMDH-type neural network algorithm, which are shown in Fig.5.

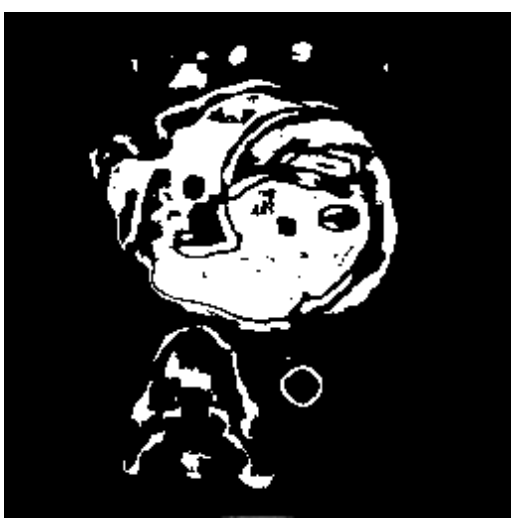

(a) $m=5$

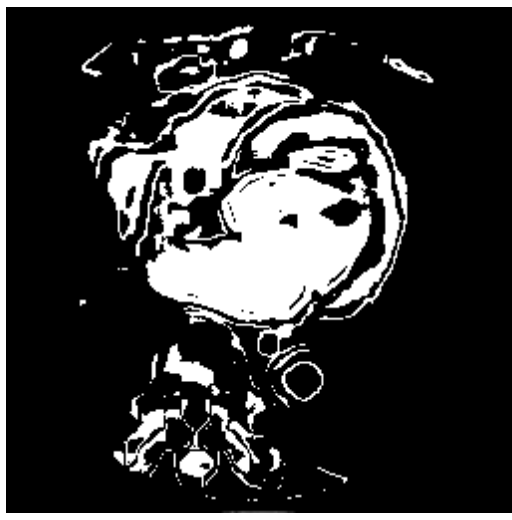

(b) $m=7$

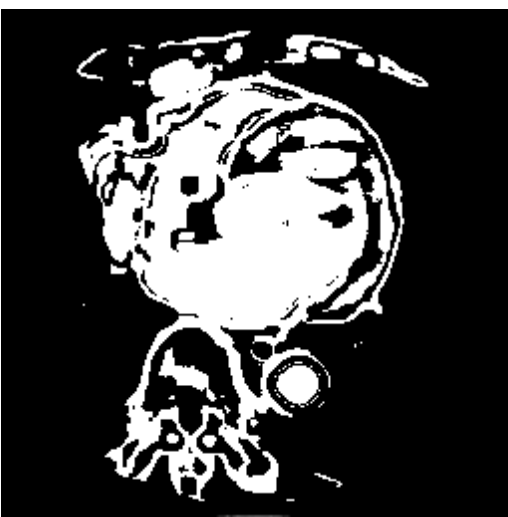

(c) $m=9$

Fig. 9 Output images of the conventional sigmoid function neural network 


\section{Conclusions}

In this paper, the deep multi-layered GMDH-type neural network algorithm was applied to the medical image recognition of heart regions and the results were compared with those of the conventional sigmoid function neural network trained using the back propagation algorithm. In this algorithm, the multicolinearity is completely eliminated and the stable and accurate prediction values are obtained. Furthermore, the optimum neural network architectures are automatically selected from three types of neural network architectures. In this study, the deep neural network architecture which had eight hidden layers, was automatically organized and the sigmoid function type neural network architecture was automatically selected so as to minimize PSS criterion. The medical image recognition results were very accurate. It was shown that the deep multi-layered GMDH-type neural network algorithm was a useful method for the medical image recognition of heart regions and this algorism was easy to be applied to the practical complex problems in which very complex neural network architectures with many hidden layers were needed.

\section{Acknowledgment}

This work was supported by (JSPS) KAKENHI 26420421.

\section{References}

1. T. Kondo, J. Ueno and S. Takao, Hybrid multi-layered GMDH-type neural network using principal component regression analysis and its application to medical image diagnosis of liver cancer, Procedia Computer Science, 22, (2013) 172-181.
2. T. Kondo, J. Ueno and S. Takao, Hybrid feedback GMDH-type neural network using principal componentregression analysis and its application to medical image diagnosis of lung cancer, ICIC Express Letters (ICIC-EX) 8 (4) (2014) 1053-1060.

3. C. Kondo, T. Kondo and S. Takao, Three-dimensional medical image analysis of the heart by the revised GMDH-type neural network self-selecting optimum neural network architecture, Artificial life and robotics, 14 (2) (2009) 123-128.

4. T. Kondo and J. Ueno, Revised GMDH-type neural network algorithm for medical image recognition and its application to 3-dimensional medical image analysis of the heart, Proceedings of the $40^{\text {th }}$ ISCIE international symposium on stochastic systems theory and its application, (2009) 148-153.

5. S. J. Farlow ed., Self-organizing methods in modeling, GMDH-type algorithm, New York: Marcel Dekker Inc., (1984)

6. A. G. Ivakhnenko, Heuristic self-organization in problems of engineering cybernetics, Automatica, 6 (2) (1970) 207-219.

7. H. Akaike, A new look at the statistical model identification, IEEE Trans. Automatic Control AC-19 (6) (1974) 716-723.

8. H. Tamura, T. Kondo, Heuristics free group method of data handling algorithm of generating optimum partial polynomials with application to air pollution prediction, Int. J. System Sci. 11 (9) (1980) 1095-1111.

9. N.R. Draper and H. Smith, Applied regression analysis, John Wiley and Sons, New York (1981). 